\title{
F-RINGS OF CONTINUOUS FUNCTIONS I
}

\author{
BARRON BRAINERD
}

1. Introduction. It is well known $(2,4)$ that the ring of all real (complex) continuous functions on a compact Hausdorff space can be characterized algebraically as a Banach algebra which satisfies certain additional intrinsic conditions. It might be expected that rings of all continuous functions on other topological spaces also have algebraic characterizations. The main purpose of this note is to discuss two such characterizations. In both cases the characterizations are given in the terms of the theory of $F$-rings (1). In one case a characterization is given for the ring of all (real) continuous functions on a generalized $P$-space, that is, a zero-dimensional topological space in which the class of open-closed sets forms a $\sigma$-algebra. A Hausdorff generalized $P$-space is a $P$-space in the terminology of (3). In the other case a theorem of Sikorski (6) is employed to give a characterization of the ring of all (real) continuous functions on an upper $\boldsymbol{\aleph}_{1}$-compact $P$-space. A $P$-space is said to be upper $\boldsymbol{\aleph}_{1}$-compact if every open covering of the space can be replaced by an at most countable subcovering.

It is remarked in a previous paper (1) that the ring of all (real) continuous functions on a $P$-space is a regular $M$-ring and hence is the ring of all $(\Omega, \mathfrak{F})$ measurable functions where $\Omega$ is the domain of the continuous function ring and $\mathfrak{F}$ is a certain distinguished $\sigma$-algebra of subsets of $\Omega$. A portion of this note is devoted to a characterization of those $\mathfrak{F}$ 's for which a topology $\mathfrak{I}$ exists such that the ring of all $(\Omega, \mathfrak{F})$-measurable functions is exactly the ring of all real functions on $\Omega$ which are continuous under the topology $\mathfrak{T}$.

The notation and definitions of (1) are used here with the exception that $M(\Omega, \mathfrak{F})$ is used to denote the regular $M$-ring of all $(\Omega, \mathfrak{F})$-measurable functions. Since the following discussion involves a multiplicity of topologies defined on a given abstract space, the notation $[\Omega, \mathfrak{T}]$ is used to designate a topological space having $\Omega$ as its set of points and $\mathfrak{T}$ as its class of open sets. $C[\Omega, \mathfrak{I}]$ designates the ring of all (real) continuous functions on $[\Omega, \mathfrak{T}]$. When there is no ambiguity the shortened forms $M(\mathfrak{F})$ and $C[\mathfrak{T}]$ are used respectively for $M(\Omega, \mathfrak{F})$ and $C[\Omega, \mathfrak{I}]$.

If $\mathfrak{A}$ is an algebra of subsets of some set $\Omega$, then $T \mathfrak{U}$ stands for the class of open sets in the topology generated by $\mathfrak{A}$, and if $[\Omega, \mathfrak{I}]$ is a topological space, $\zeta \mathfrak{I}$ stands for the class of all open-closed sets in $[\Omega, \mathfrak{T}]$. If $p \in \Omega$, then $\mathfrak{M}_{p}$ designates the set $\{A \in \mathfrak{H} ! p \notin A\}$ which is clearly a maximal ideal of $\mathfrak{A}$.

Received February 13, 1958. The author wishes to thank B. Banaschewski for his helpful discussions of some of the results of this paper. 
If $\mathfrak{D}$ is a collection of sets, then $\cap \mathfrak{D}$ and $\cup \mathfrak{D}$ designate respectively the intersection and union of the elements in $\mathfrak{D}$.

2. Generalized $P$-spaces and $\sigma$-algebras. Let $\Omega$ be a set which contains at least two points and let $\mathfrak{F}$ be a $\sigma$-algebra of subsets of $\Omega$. If $\mathfrak{T}$ is a topology on $\Omega$ such that $M(\mathfrak{F})$ is the ring of all continuous functions on $[\Omega, \mathfrak{T}]$, then $\mathfrak{F} \subseteq \mathfrak{I}$ and hence $T \mathfrak{F} \subseteq \mathfrak{I}$. It is a matter of direct verification to show that in general $[\Omega, T \mathfrak{F}]$ is a generalized $P$-space and $M(\mathfrak{F}) \subseteq C[T \mathfrak{F}]$. Thus if there is a $\mathfrak{I}$ such that $M(\mathfrak{F})=C[\mathfrak{I}]$, then $M(\mathfrak{F})=C[T \mathfrak{F}]=C[\mathfrak{I}]$. Hence the problem of determining those $\mathfrak{F}$ for which there exists a $\mathfrak{I}$ such that $C[\mathfrak{T}]=M(\mathfrak{F})$ is reduced to the problem of determining those $\mathfrak{F}$ for which $C[T \mathfrak{F}]=M(\mathfrak{F})$.

THEOREM 1. If $\mathfrak{F}$ is a $\sigma$-algebra of subsets of $\Omega$, then the following statements are equivalent:

(i) $C[T \mathfrak{F}]=M(\mathfrak{F})$,

(ii) $\zeta T \mathfrak{F}=\mathfrak{F}$,

(iii) If $\mathfrak{B}$ is an ideal of $\mathfrak{F}$ with properties:

(a) $\mathfrak{B}=\cap\left\{\mathfrak{M}_{p} \mid \mathfrak{M}_{p} \supseteq \mathfrak{B}\right\}$,

(b) $\mathfrak{M}_{p} \supseteq \mathfrak{B} \Longrightarrow$ there is a principal ideal $\mathfrak{S}_{p}$ such that $\mathfrak{B} \subseteq \mathfrak{\Im}_{p} \subseteq \mathfrak{M}_{p}$, then $\mathfrak{B}$ is principal.

Proof. (i) $\Rightarrow$ (ii): If $A$ is an open-closed set in the topology $T \mathfrak{F}$, then $\chi_{A}$ is continuous and hence belongs to $C[T \mathfrak{F}]=M(\mathfrak{F})$. Thus it is clear that $A=\left\{p \in \Omega \mid \chi_{A} \geqslant \frac{1}{2}\right\}$ belongs to $\mathfrak{F}$. Therefore $\zeta T \mathfrak{F} \subseteq \mathfrak{F}$. Obviously $\mathfrak{F} \subseteq \zeta T \mathfrak{F}$.

(ii) $\Rightarrow$ (iii): If $\mathfrak{B}$ has property (iiia), then $\mathfrak{B}=\{A \in \mathfrak{F} \mid A \subseteq D\}$ where $D=\cup \mathfrak{B}$. Indeed, suppose $A \subseteq D$. Then if $\mathfrak{M}_{p} \supseteq \mathfrak{B}$, it follows that $p \notin D$, and therefore $\mathfrak{M}_{p} \supseteq \mathfrak{B}$ implies that $A \in \mathfrak{M}_{p}$. Thus $A \in \cap\left\{\mathfrak{M}_{p} \mid \mathfrak{M}_{p} \supseteq \mathfrak{B}\right\}=\mathfrak{B}$.

If $\mathfrak{B}$ satisfies both (iiia) and (iiib), then $\mathfrak{B}$ is principal. This is proved by showing that $D$ is open-closed and then noting that $D \in \mathfrak{B}$. Let $p \notin D$. Then $\mathfrak{M}_{p} \supseteq \mathfrak{B}$ and there is a principal ideal $\mathfrak{\Im}_{p}$ such that $\mathfrak{B} \subseteq \mathfrak{\Im}_{p} \subseteq \mathfrak{M}_{p}$. If $I_{p}$ is the generator of $\Im_{p}$, then $p \notin I_{p}$ and $B \subseteq I_{p}$ for all $B \in \mathfrak{B}$. Thus $D \subseteq I_{p}$ and $p \in \Omega-I_{p} \in \mathfrak{F}$. Therefore $D$ is open-closed and belongs to $\mathfrak{F}$. If $D \notin \mathfrak{B}$, then for some $\mathfrak{M}_{p} \supseteq \mathfrak{B}$ we have $p \in D$ which leads to a contradiction because $\mathfrak{M}_{p} \supseteq \mathfrak{B}$ implies $p \notin D$. This proves that $\mathfrak{B}$ is the principal ideal generated by $D$.

(iii) $\Rightarrow$ (ii): Let $D$ be an open-closed set in the topology $T \mathfrak{F}$. The ideal $\mathfrak{D}=\{A \in \mathfrak{F} \mid A \subseteq D\}$ satisfies (iiia). Indeed, if $A \in \cap\left\{\mathfrak{M}_{p} \mid \mathfrak{M}_{p} \supseteq \mathfrak{D}\right\}$, then $p \notin A$ for all $\mathfrak{M}_{p} \supseteq \mathfrak{D}$. Since $\mathfrak{M}_{p} \supseteq \mathfrak{D}$ is equivalent to $p \notin D$, it then follows that $A \in \cap\left\{\mathfrak{M}_{p} \mid \mathfrak{M}_{p} \supseteq \mathfrak{D}\right\}$ implies $p \notin A$ for all $p \notin D$ which in turn implies $A \subseteq D$. Thus $\cap\left\{\mathfrak{M}_{p} \mid \mathfrak{M}_{p} \supseteq \mathfrak{D}\right\}=\mathfrak{D}$.

If $p \notin D$, then there is a $B \in \mathfrak{F}$ such that $p \in B$ and $B \cap D=\phi$, because $D$ is closed. Hence $\Omega-B \supseteq D$ and the principal ideal $\Im_{p}$ generated by $\Omega-B$ contains $\mathfrak{D}$. Therefore if $\mathfrak{M}_{p} \supseteq \mathfrak{D}$, there is a principal ideal $\Im_{p}$ such that $\mathfrak{D} \subseteq \mathfrak{\Im}_{p} \subseteq \mathfrak{M}_{p}$. Thus $\mathfrak{D}$ satisfies (iiib) and $\mathfrak{D}$ is principal. 
Now $\cup \mathfrak{D}=D$, because $D$ is open. Since $\mathfrak{D}$ is principal, $D$ is its generator; hence $D \in \mathfrak{F}$ and $\mathfrak{F}=\zeta T \mathfrak{F}$.

(ii) $\Rightarrow$ (i): Since $M(\mathfrak{F}) \subseteq C[T \mathfrak{F}]$, statement (i) is established if we show that $C[T \mathfrak{F}] \subseteq M(\mathfrak{F})$. Suppose $\mathfrak{g} \in C[T \mathfrak{F}]$. Then as in (3, Theorem 5.3), $\mathrm{g}^{-1}(\alpha)=\{p \in \Omega \mid \mathfrak{g}(p)=\alpha\}$ is an open set for any real $\alpha$ (because countable intersections of sets in $T \mathfrak{F}$ belong to $T \mathfrak{F})$ and hence

$$
\{p \in \Omega \mid g(p) \leqslant \lambda\}=\bigcup\left\{\mathfrak{g}^{-1}(\alpha) \mid \alpha \leqslant \lambda\right\} \in \zeta T \mathfrak{F}=\mathfrak{F}
$$

Therefore $\mathfrak{g} \in M(\mathfrak{F})$.

As an example of an $\mathfrak{F}$ for which $C[T \mathfrak{F}] \neq M(\mathfrak{F})$, let $\mathfrak{F}$ be the class of all Borel sets on the real line $E$. It is clear that $T \mathfrak{F}$ is the discrete topology on $E$ and that $C[T \mathfrak{F}]$ is the ring of all real functions on $E$.

In Theorem 1 the proof that (ii) $\Leftrightarrow$ (iii) does not depend on the hypothesis that $\mathfrak{F}$ is an $\sigma$-algebra. Therefore the following more general result can be obtained:

Corollary 1. Let $\mathfrak{A}$ be an algebra of subsets of $\Omega$. Then $\mathfrak{A}$ satisfies condition (ii) if and only if $(\Omega, \mathfrak{H})$ satisfies condition (iii).

The following corollary, useful in the sequel, is a direct consequence of Theorem 1:

Corollary 2. If $[\Omega, \mathfrak{T}]$ is a generalized P-space, then $C[\Omega, \mathfrak{T}]=M(\Omega, \zeta \mathfrak{T})$.

Indeed, in a generalized $P$-space, $T \zeta \mathfrak{I}=\mathfrak{I}$ and $\zeta T \zeta \mathfrak{I}=\zeta \mathfrak{T}$. Hence by Theorem 1 the equation $C[\Omega, \mathfrak{I}]=M(\Omega, \zeta \mathfrak{I})$ is valid.

3. A maximal ideal theorem. Before consideration of the characterizations, a theorem and a corollary concerning maximal ideals are proved. These make clear the relationship between the concepts of closed maximal ideal and real maximal ideal for regular $F$-rings.

Theorem 3. Let $R$ be a regular F-ring. If a maximal ideal $\mathfrak{M}$ of $R$ is real, it is also closed.

For definitions of real and closed see (1). In order to prove Theorem 3 the following lemma is employed.

LEMMA 1. If $\left\{e_{n}\right\}$ is an orthogonal sequence of idempotents of $R$ such that $\mathrm{V}_{n=1}^{\infty} e_{n}=1$ and if $\left\{\alpha_{n}\right\}$ is a sequence of real numbers such that $0 \leqslant \epsilon \leqslant \alpha_{n}$ for all integers $n \geqslant 1$, then $\mathrm{V}_{n=1}^{\infty} \alpha_{n} e_{n} \in R$.

Proof. The element $b=\mathrm{V}_{n:=1}^{\infty} 1 / \alpha_{n} e_{n}$ belongs to $R$. From the equation

$$
\bar{e}_{b}=\mathrm{V}_{m=1}^{\infty}(1 \wedge m b)=\mathrm{V}_{m=1}^{\infty}\left[1 \wedge m\left(\mathrm{~V}_{n=1}^{\infty} e_{n} / \alpha_{n}\right]\right.
$$

we deduce by the distributivity of the lattice operations that $\bar{e}_{b}=\mathrm{V}_{n=1}^{\infty} e_{n}=1$. Hence the regularity of $R$ implies (1, Theorem 2) that $b^{-1} \in R$. Clearly

$$
e_{m}=b b^{-1} e_{m}=\left(\mathrm{V}_{n=1}^{\infty} e_{n} / \alpha_{n}\right) b^{-1} e_{m}=e_{n} / \alpha_{n} b^{-1},
$$


so $\alpha_{n} e_{n}=b^{-1} e$ for $n \geqslant 1$. Therefore, since $b^{-1}=\left(\mathrm{V}_{n=1}^{\infty} e_{n}\right) b^{-1}=\mathrm{V}_{n=1}^{\infty}\left(e_{n} b^{-1}\right)$, it follows that $b^{-1}=\mathrm{V}_{n=1}^{\infty} \alpha_{n} e_{n}$.

Proof of Theorem 3. Suppose $\mathfrak{M}$ is not closed. Then there is a sequence $\left\{e_{n}\right\}$ of orthogonal idempotents such that $\mathrm{V}_{n=1}^{\infty} e_{n}=1$ and $e_{n} \in \mathfrak{M}$ for each integer $n \geqslant 1$. Indeed, there is a sequence $\left\{b_{n}\right\}$ of elements of $\mathfrak{M}$, such that $b_{n} \geqslant 0$ for all integers $n \geqslant 1$ and $b=\mathrm{V}_{n=1}^{\infty} b_{n} \in R$ while $b \notin \mathfrak{M}$. Since

$$
\mathrm{V}_{n=1}^{\infty} \bar{e}_{b_{n}}=\mathrm{V}_{n=1}^{\infty} \mathrm{V}_{m=1}^{\infty}\left[1 \wedge m b_{n}\right]=\mathrm{V}_{m=1}^{\infty}\left[1 \wedge m \mathrm{~V}_{n=1}^{\infty} b_{n}\right]=\bar{e}_{b}
$$

and since $x \in \mathfrak{M}$ if and only if $\bar{e}_{x} \in \mathfrak{M}$, it follows that $\bar{e}_{b}$ does not belong to $\mathfrak{M}$. Therefore $e_{0}=1-\bar{e}_{b} \in \mathfrak{M}$. Let

$$
e_{n}=\left[e_{0} \vee\left(\mathrm{V}_{m=1}^{n} \bar{e}_{b_{m}}\right)\right] \wedge\left\{1-\left[e_{0} \vee\left(\mathrm{V}_{m=1}^{n-1} \bar{e}_{b_{m}}\right)\right]\right\}
$$

for $n \geqslant 1$. Then it can be shown that $\left\{e_{0}, e_{1}, \ldots\right\}$ is an orthogonal sequence of idempotents such that $\mathrm{V}_{n=0}^{\infty} e_{n}=1$, while $e_{n} \in \mathfrak{M}$ for each integer $n \geqslant 0$.

If $\left\{e_{n}\right\}$ is a sequence of orthogonal idempotents in $\mathfrak{M}$ such that $\mathrm{V}_{n=0}{ }^{\infty} e_{n}=1$, then by Lemma $1, d=\mathrm{V}_{n=0}{ }^{\infty}(n+1) e_{n}$ belongs to $R$ and has an inverse $d^{-1} \in R$. If $m$ is a natural number, then

$$
d-m \cdot 1=\sum_{n=0}^{m-1}(n+1-m) e_{n}+\mathrm{V}_{n=m}^{\infty}(n+1-m) e_{n}
$$

belongs to $R$. Since $\sum_{n=0}{ }^{m-1}(n+1-m) e_{n}$ belongs to $\mathfrak{M}$ for all $m \geqslant 1$, it follows that the non-negative element $\mathrm{V}_{n=m}^{\infty}(n+1-m) e_{n}$ and the element $d-m \cdot 1$ have the same image under the natural homomorphism of $R$ onto $R-\mathfrak{M}$. This homomorphism preserves order, and hence $d(\mathfrak{M})-m \cdot 1 \geqslant 0$ for all choices of $m \geqslant 1$. Therefore $R-\mathfrak{M}$ cannot be the real field.

COROLlaRy. If $R$ is a regular F-ring, then a maximal ideal is real if and only if it is closed.

Proof. This follows from Theorem 3 and (1, Theorem 5).

4. Characterizations. In this section, when $R$ is a regular $F$-ring $B(R)$ is used to designate the collection of idempotents of $R$. It is shown in (1) that $B(R)$ is a $\sigma$-complete Boolean algebra with respect to the lattice operations of $R$.

Let $G$ be either a regular $F$-ring or a $\sigma$-complete Boolean algebra, and let $\Omega$ stand for the class of all closed maximal ideals of $G$. Consider the following conditions:

( $\alpha$ ) If $\mathfrak{B}$ is an ideal of $G$ of the form $\mathfrak{B}=\cap\{\mathfrak{M} \in \Omega \mid \mathfrak{M} \supseteq \mathfrak{B}\}$ and if for each $\mathfrak{M} \in \Omega$ such that $\mathfrak{M} \supseteq \mathfrak{B}$ there exists a principal ideal $\mathfrak{Y}$ which satisfies the relation $\mathfrak{B} \subseteq \mathfrak{Y} \subseteq \mathfrak{M}$, then $\mathfrak{B}$ is a principal ideal.

$(\beta)$ Every proper closed ideal of $G$ is a subset of some closed maximal ideal of $G$.

Lemma 2. Let $R$ be a regular F-ring. The ideal lattices of $R$ and $B(R)$ are isomorphic (under $J \rightarrow J \cap B(R)$ and its inverse $J \rightarrow R J$ ) such that principal ideals correspond to principal ideals and closed ideals to closed ideals. 
Proof. The isomorphism of the ideal lattices and the result that principal ideals correspond to principal ideals are trivial consequences of (5, Theorem $5)$. It remains only to prove that closed ideals correspond to closed ideals. An ideal $Q$ of $R$ is closed if and only if for a sequence $\left\{f_{n}\right\}$ of non-negative elements of $Q$, the existence of $f=\mathrm{V}_{n=1}^{\infty} f_{n}$ in $R$ implies $f \in Q$. Suppose $J$ is a closed ideal of $B(R)$. If $\left\{f_{n}\right\}$ is a sequence of non-negative elements of $R J$, then

$$
\bar{e}_{f_{n}} \in J, \quad n \geqslant 1,
$$

and hence

$$
\mathrm{V}_{n=1}^{\infty} \bar{e}_{f_{n}} \in J \text {. }
$$

If $f=\mathrm{V}_{n=1}^{\infty} f_{n}$ exists in $R$, then as in the proof of Theorem 3 it can be shown that

$$
\bar{e}_{f}=\mathrm{V}_{n=1}^{\infty} \bar{e}_{f_{n}} .
$$

Hence $\bar{e}_{f} \in J$ and $f=f \bar{e}_{f} \in R . J$. Therefore $R J$ is also closed. If $Q$ is a closed ideal of $R$, then trivially $Q \cap B(R)$ is a closed ideal of $B(R)$.

The following useful corollary is an immediate consequence of Lemma 2 .

Corollary. If $R$ is a regular F-ring, then $R$ satisfies condition $\alpha(\beta)$ if and only if $B(R)$ satisfies condition $\alpha(\beta)$.

It is now possible to characterize the ring of all continuous (real) functions on a generalized $P$-space.

TheORem 4. A regular F-ring $R$ is isomorphic to the ring of all continuous functions on a generalized $P$-space if and only if $R$ is an $M$-ring which satisfies condition $\alpha$.

Proof. Let $R$ be an $M$-ring which satisfies condition $\alpha$. From Theorems 7 and 8 of (3) it follows that there is a correspondence $\phi: x \rightarrow \hat{x}$ which maps $x$ onto a real function $\hat{x}$ defined on $\Omega$. At each $\mathfrak{M} \in \Omega$ the value of $\hat{x}$ is the image of $x$ under the homomorphism with kernel $\mathfrak{M}$. This correspondence $\phi$ is an isomorphism of $R$ onto an $M$-ring $M(\Omega, \mathfrak{F})$, and under it the Boolean algebra $B(R)$ is mapped isomorphically onto the set of characteristic functions of elements in the $\sigma$-algebra $\mathfrak{F}$. In addition $B(R) \cong \mathfrak{F}$ under the correspondence $x \rightarrow\{\mathfrak{M} \in \Omega \mid \hat{x}(\mathfrak{M})=1\}, \quad[\Omega, T \mathfrak{F}]$ is a generalized $P$-space (in particular since $[\Omega, T \mathfrak{F}]$ is Hausdorff it is a $P$-space), and finally the ideal lattices of $R$ and $B(R)$ are isomorphic (Lemma 2). Therefore if $R$ satisfies $\alpha$, then $\mathfrak{F}$ satisfies $\alpha$ also and from Theorem 1 it follows that $M(\Omega, \mathfrak{F})=C[\Omega, T \mathfrak{F}]$.

Conversely, if $\left[\Omega^{*}, \mathfrak{I}\right]$ is a generalized $P$-space, then (Corollary 2 , Theorem 1) $C\left[\Omega^{*}, \mathfrak{I}\right]=M\left(\Omega^{*}, \zeta \mathfrak{I}\right)$. Therefore $C\left[\Omega^{*}, \mathfrak{T}\right]$ is an $M$-ring and the $\sigma$-algebra $\zeta \mathfrak{I}$ satisfies condition (iii) of Theorem 1. Suppose $\mathfrak{B}$ is an ideal of $\zeta \mathfrak{I}$ which satisfies the hypothesis of condition $\alpha$. Let $\mathfrak{B}^{*}=\left\{\mathfrak{M}_{p} \mid p \in \Omega^{*}, \mathfrak{M}_{p} \supseteq \mathfrak{B}\right\}$. Clearly $\mathfrak{B} \subseteq \mathfrak{B}^{*}$ and hence $B=\cup \mathfrak{B} \subseteq B^{*}=\cup \mathfrak{B}^{*}$. Since $p \notin B$ if and only if $\mathfrak{M}_{p} \supseteq \mathfrak{B}$ and $p \notin B^{*}$ if and only if $\mathfrak{M}_{p} \supseteq \mathfrak{B}^{*}$, it follows that $B=B^{*}$. Suppose $A \in \mathfrak{B}^{*}-\mathfrak{B}$. Then there is a closed maximal ideal $\mathfrak{M}$ such that $A \notin \mathfrak{M}$ 
and $\mathfrak{M} \supseteq \mathfrak{B}$. There is a principal ideal $\mathfrak{S}$ with generator $I$ such that $\mathfrak{M} \supseteq \mathfrak{J}$ $\supseteq \mathfrak{B}$. Hence $I \supseteq B \supseteq A$. This contradicts the statement $A \notin \mathfrak{M}$; therefore $\mathfrak{B}^{*}=\mathfrak{B}$. From Theorem 1 it follows that $\mathfrak{B}$ is principal and hence $\zeta \mathfrak{T}$ satisfies condition $\alpha$. Therefore both $B\left(C\left[\Omega^{*}, \mathfrak{T}\right]\right)$ and $C\left[\Omega^{*}, \mathfrak{I}\right]$ satisfy condition $\alpha$.

For the characterization of the ring of all continuous functions on an upper $\boldsymbol{\aleph}_{1}$-compact $P$-space the following theorem is employed.

Theorem 5 (Sikorski). If $\Omega$ is the set of all closed maximal ideals of a $\sigma$-complete Boolean algebra $B$, then the following statements are equivalent:

(i) There exists a topology $\mathfrak{I}$ on $\Omega$ such that the correspondence

$$
a \rightarrow\{\mathfrak{M} \in \Omega \mid a \notin \mathfrak{M}\}
$$

is an isomorphism of $B$ onto the $\sigma$-algebra of open-closed subsets of the upper $\aleph_{1}$-compact P-space $[\Omega, \mathfrak{T}]$.

(ii) $B$ satisfies condition $\beta$.

This theorem is a restatement of (6, Theorem xviii) in terms of the symbolism of the present paper.

The following theorem constitutes the characterization.

Theorem 6. Let $R$ be a regular F-ring. A necessary and sufficient condition for $R$ to be isomorphic to $C[\Omega, \mathfrak{T}]$ for some upper $\boldsymbol{\aleph}_{1}$-compact $P$-space $[\Omega, \mathfrak{T}]$ is that $R$ satisfy condition $\beta$.

Proof. Assume $R$ satisfies $\beta$. Since principal ideals of $R$ are closed, every principal ideal is a subset of a closed maximal ideal. Therefore $R$ is an $M$-ring. In addition (see proof of Theorem 4 and (3, Theorems 7 and 8$)$ ), $R$ is isomorphic to $M(\Omega, \mathfrak{F})$ where the isomorphism maps $B(R)$ onto the $\sigma$-algebra of characteristic functions on $\mathfrak{F}$ and $\mathfrak{F} \cong B(R)$. Therefore (Lemma 2 ) $\mathfrak{F}$ satisfies $\beta$ and since closed maximal ideals of $\mathfrak{F}$ are of the form $\mathfrak{M}_{p}$ for $p \in \Omega$ we deduce from Theorem 5 that $\mathfrak{F}$ is the class of open-closed sets for an upper $\boldsymbol{\aleph}_{1}$-compact $P$-topology $\mathfrak{T}$ on $\Omega$. Thus $\mathfrak{F}=\zeta \mathfrak{T}$ and from Corollary 2, Theorem 1 it follows that $C[\Omega, \mathfrak{I}]=M(\Omega, \mathfrak{F}) \cong R$.

Assume $\left[\Omega^{*}, \mathfrak{I}\right]$ is an upper $\boldsymbol{\aleph}_{1}$-compact $P$-space. The upper $\boldsymbol{\aleph}_{1}$-compactness implies that each closed maximal ideal of $\zeta \mathfrak{T}$ is fixed. Therefore (Theorem 5) the $\sigma$-algebra $\zeta \mathfrak{I}$ satisfies condition $\beta$; hence $M(\Omega, \zeta \mathfrak{I})$ satisfies $\beta$ as well. Finally by Corollary 2 , Theorem 1 we have $C[\Omega, \mathfrak{T}]=M(\Omega, \zeta \mathfrak{T})$. 


\section{REFERENCES}

1. B. Brainerd, On a class of lattice-ordered rings, Proc. Amer. Math. Soc., 8 (1957), 673-83.

2. I. Gelfand, Normierte Ringe, Rec. Math. (Mat. Sbornik) N.S., 9, 51 (1941), 3-24.

3. L. Gillman and M. Henriksen, Concerning rings of continuous functions, Trans. Amer. Math. Soc., 77 (1954), 340-62.

4. E. Hewitt, Rings of real-valued continuous functions I, Trans. Amer. Math. Soc., 64 (1948), 45-99.

5. D. R. Morrison, Bi-regular rings and the ideal lattice isomorphisms, Proc. Amer. Math. Soc., 16 (1955), 46-9.

6. R. Sikorski, Remarks on some topological spaces of high power, Fund. Math., 37 (1950), $125-36$.

The University of Western Ontario

and

Summer Research Institute of the

Canadian Mathematical Congress 